\title{
MORE TRANSIENT RESULTS FOR GENERALISED STATE-DEPENDENT ERLANGIAN QUEUES
}

\author{
B. W. CONOLLY, ${ }^{*}$ Chelsea College, London
}

STATE-DEPENDENT QUEUES; GENERALIZED BIRTH AND DEATH PROCESSES; TIME-DEPENDENT RESULTS

Setting examination questions is often a nuisance, but occasionally it can be a productive exercise. Recently it enabled some gaps in Conolly (1975) to be filled. In that paper single-server queueing systems driven by $\left(\lambda_{n}, \mu_{n}\right)$ arrival and service mechanisms were discussed. These basically exponential mechanisms fluctuate with system state $n$. Three models were considered.

Model A, having $\lambda_{n}=\lambda, \mu_{n}=\mu n(\lambda, \mu>0)$, embodies the notion of server cooperation and it possesses compound Poisson state probabilities

$$
p_{n}(t)=e^{-\rho \Lambda(t)}\{\rho \Lambda(t)\}^{n} / n !,
$$

where $\rho=\lambda / \mu, \Lambda(t)=1-e^{-\mu t}$, for all non-negative state sizes $n$, given that $p_{0}(0)=1$. This system mimics $M / M / \infty$.

Model B has $\lambda_{n}=\lambda /(n+1), \mu_{n}=\mu(\lambda, \mu>0)$. This embodies customer reluctance or discouragement. Although the asymptotic form of $p_{n}(t)$ as $t \rightarrow \infty$ is identical with that of Model A, the time-dependent behaviour is less elegant (Natvig (1974), Van Doorn (1981)).

Model C incorporates the mechanisms of A and B. Thus $\lambda_{n}=\lambda /(n+1), \mu_{n}=$ $\mu n(\lambda, \mu>0)$. Service is cooperative and customers reluctant. The asymptotic form of the state probabilities is easily obtained, namely, as $t \rightarrow \infty, p_{n}(t) \rightarrow \bar{p}_{n}$, where

$$
\bar{p}_{n}=\frac{\rho^{n}}{n ! n ! I_{0}\left(2 \rho^{\frac{1}{2}}\right)} \text {. }
$$

Here and in the following

$$
I_{n}(x)=\sum_{j=0}^{\infty} \frac{(x / 2)^{n+2 j}}{j !(j+n) !} \quad(n \text { integer })
$$

is the modified Bessel function of the first kind with argument $x$ and order $n$. It satisfies the differential equation

$$
x^{2} y^{\prime \prime}+x y^{\prime}-\left(x^{2}+n^{2}\right)=0 .
$$

The examination exercises referred to enable it to be stated that $p_{n}(t)$, with initial

Received 18 April 1983; revision received 22 June 1983.

* Postal address: Department of Mathematics, Chelsea College, University of London, 552 Kings Road, London SW10 0UA. 
value $p_{0}(0)=1$, is generated by $P(x, t)=\sum x^{n} p_{n}(t)$, where

$$
P(x, t)=\frac{I_{0}\left(2(\rho x)^{\frac{1}{2}}\right)}{I_{0}\left[2\left\{\rho\left(1+(x-1) e^{-\mu t}\right)\right\}^{\frac{1}{2}}\right]} .
$$

This result is obtained by showing in the usual way that $P(x, t)$ satisfies

where

$$
x \frac{\partial P(x, t)}{\partial t}+\mu x(x-1) \frac{\partial P(x, t)}{\partial x}=\lambda(x-1) G(x, t)
$$

$$
G(x, t)=\int_{0}^{x} P(y, t) d y
$$

and using Lagrange's method to obtain the first obvious integral $u=(x-1) e^{-\mu t}$ of the characteristics. The other pair of equations can be written as

$$
\frac{\partial^{2} G(x, t)}{\partial x^{2}}-\frac{\rho G(x, t)}{x}=0
$$

which, by transformation of the Bessel function differential equation, can be seen to be satisfied by

$$
G(x, t)=x^{\frac{1}{2}} I_{1}\left(2(\rho x)^{\frac{1}{2}}\right) .
$$

This gives for the second integral

$$
\frac{P(x, t)}{v}=\frac{d}{d x}\left[x^{\frac{1}{2}} I_{1}\left(2(\rho x)^{\frac{1}{2}}\right)\right],
$$

where $v$ is the integration constant. Since $I_{1}(x)=I_{0}^{\prime}(x)$, and by use of the fundamental differential equation, we can express the general solution $v=F(u)$ in the form

$$
P(x, t)=\rho^{\frac{1}{2}} I_{0}\left(2(\rho x)^{\frac{1}{2}}\right) F\left[(x-1) e^{-\mu t}\right],
$$

where $F$ is a function whose form is revealed by the initial condition $P(x, 0)=1$. Thus

and (1) follows.

$$
F(x)=\left[\rho^{\frac{1}{2}} I_{0}\left\{2(\rho(x+1))^{\frac{1}{2}}\right\}\right]^{-1},
$$

In particular,

$$
\begin{gathered}
p_{0}(t)=\frac{1}{I_{0}\left(2(\rho \Lambda(t))^{\frac{1}{2}}\right)}, \\
p_{1}(t)=\frac{\rho}{I_{0}\left(2(\rho \Lambda(t))^{\frac{1}{2}}\right)}\left[\Lambda(t)+e^{-\mu t} \frac{I_{2}\left(2(\rho \Lambda(t))^{\frac{1}{2}}\right.}{I_{0}\left(2(\rho \Lambda(t))^{\frac{1}{2}}\right)}\right],
\end{gathered}
$$

and, for the record, the mean $m(t)$ is

$$
m(t)=\frac{\rho^{\frac{1}{2}} I_{1}\left(2 \rho^{\frac{1}{2}}\right)}{I_{0}\left(2 \rho^{\frac{1}{2}}\right)} \Lambda(t)
$$

Conolly (1975) mentions an even more efficient mechanism than Model C. This is characterised by $\lambda_{n}=\lambda /(2 n+1), \mu_{n}=2 \mu n(\lambda, \mu>0)$ and, under the same initial condition $p_{0}(0)=1$, this can be dealt with similarly. A specimen result is

$$
p_{0}(t)=\frac{1}{\cosh \left(\rho\left(1-e^{-2 \mu t}\right)\right)^{\frac{1}{2}}} .
$$

No doubt future generations of students will produce others. 


\section{References}

CONOLLY, B. W. (1975) Generalized state-dependent Erlangian queues: speculations about calculating measures of effectiveness. J. Appl. Prob. 12, 358-363.

NATVIG, B. (1974) On the transient state probabilities for a queueing model where potential customers are discouraged by queue length. J. Appl. Prob. 11, 345-354.

VAN DOORN, E. A. (1981) The transient state probabilities for a queueing model where potential customers are discouraged by queue length. J. Appl. Prob. 18, 499-506. 